\title{
Edge Preserving Image Coding For High Resolution Image Representation
}

\author{
M. Nagaraju Naik ${ }^{1}$, K. Kumar Naik ${ }^{2}$, Dr. P. Rajesh Kumar ${ }^{3}$, \\ ${ }^{1}$ Associate Professor, Dept. of ECE, MIST, Hyderabad, A P, India, \\ nagraju.naik@gmail.com \\ ${ }^{2}$ Associate Professor, Dept. of ECE, ANITS, Visakhapatnam, A.P., India, \\ kumarvtr@gmail.com \\ ${ }^{3}$ Associate Professor, Dept. of ECE, A.U. College of Engineering (Autonomous), \\ Visakhapatnam, A P, India, \\ rajeshauce@gmail.com
}

\begin{abstract}
Image coding for high resolution representation, with projection edge smoothening is been proposed in this work. In past works it is observed that image projection in larger dimension carried out by image interpolation results in high stretching effects at bounding regions of the objects in an image. Though focus is made towards achieving high quality accuracy in image projection very less work is made towards preserving the edge regions. in this paper a approach towards preserving edge at the bounding edge is been focused for retaining image quality in Higher Projection grids for low dimensional images.
\end{abstract}

\section{Keywords}

Edge preserving, high resolution, Representation, Interpolation junction distortion.

\section{Introduction}

The objective of image interpolation [2] is to obtain high resolution images from low resolution inputs. It is applicable in video communication, object identification, HDTV image compression etc.

The generation of low-resolution images can be modeled as a combination of both smoothing and down-sampling by low quality sensors. Super resolution is an inverse problem for this generation, it is indeterminations due to lack of information loss.

One criteria of solving this inverse problem is minimizing the reconstruction error. Various methods were proposed in the literature to regularize the inverse problem. Two of the most extensively explored image modeling approach is the image smoothness approach and the edge smoothness approach. To preserve edge sharpness, [3, 4] is proposed to prevent cross-edge interpolation. However, locating high precision edge position itself is a non-trivial task. Level-

DOI : $10.5121 /$ acij.2012.3206 
set [5] and multiple scale tensor voting [6] methods are explored to get smooth edges. Preserving smoothness edge prior on large neighborhood is proposed [7]. A soft edge smoothness term can measure the average length of discrete level lines to produce smooth soft edges. Methods based on image modeling are more efficient. One critical issue is how to handle image edges in a satisfactory way. Simple interpolation tends to produce blurry results, while edge preserving techniques may remove image details in regions without strong edges. Where in a backpropagation approach [1] was been proposed, the error coding at the edge preservation was not achieved, and the ringing artifacts are observed more dominant. To achieve the objective of efficient image projection preserving image edge in this paper a feedback model for edge preserved coding based on adaptive -bilateral filtration is been suggested.

\section{Image Projection}

, an open-ended research problem is Super resolution still hasn't made its place in lot of textbooks on Video Processing. But, hopefully we will soon see Super resolution algorithms being implemented in digital cameras as they offer a wide variety of quality enhancement for videos. Super resolution, technique is on the most basic level, which deals with construction of a high-resolution image given less amount of low-resolution image information with some motion between them. This process examines one of the simple and faster algorithms on Super resolution.

As mentioned earlier, given a bunch of LR images, Superresolution involves two steps:

- Image Registration

- Projecting LR image values onto high-resolution grid

Most of the papers on Superresolution try to solve these two problems. Though their approaching methods are different but the end goal is same. Motion estimation is used to estimate the pixel positions of the three images with respect to the 1 image. Pixel values can take any real integer. Once this information is calculated accurately, then it is possible to project on a desired high-resolution grid. The generation process of HR image can be modeled by a combination of the blur effect (due to the atmosphere, the object/camera motion, and the sensor) and the up-sampling operation. By simplifying the blur effect with a single filter $\mathrm{g}$ for the entire image, the generation process can be formulated as follows,

$I_{h}=\operatorname{conv}\left(I_{l}, g\right)$, where $I_{h}$ and $I_{l}$ are the HR and LR images respectively,

The difference between the LR input image and the synthesized LR image gives reconstruction error of HR image. During the projection of image the aliasing and stretching effect are observed and these effects are predominantly been observed at the low frequency content basically at the edge regions. These effects introduce degradation in visual quality and need to be removed.

\section{Bilateral Image Projection}

A non-linear filtering technique [11] the space domain and the feature domain gives the combined information in the filtering process. It can be represented by the following equation

$$
\mathbf{h}(x)=\frac{1}{k(x)} \sum_{y} \mathbf{I}(y) c(x, y) s(\mathbf{I}(x), \mathbf{I}(y))
$$


where $\mathrm{I}$ and $\mathrm{h}$ are the input and output images respectively, $\mathrm{x}$ and $\mathrm{y}$ are pixel positions over the image grid, $\mathrm{c}(\mathrm{x}, \mathrm{y})$ and $\mathrm{s}(\mathrm{I}(\mathrm{x}), \mathrm{I}(\mathrm{y}))$ measure the spatial and photometric affinity between pixel $\mathrm{x}$ and pixel y respectively, and

$$
k(x)=\sum_{y} c(x, y) s(\mathbf{I}(x), \mathbf{I}(y))
$$

is the normalization factor at pixel $\mathrm{x}$. The functions $\mathrm{c}(\cdot)$ and $\mathrm{s}(\cdot)$ are usually chosen as follows

$$
\begin{aligned}
& c(x, y)=\exp \left(\frac{-\| x-y||_{2}^{2}}{2 \sigma_{c}^{2}}\right), \\
& s(u, v)=\exp \left(\frac{-\| u-\left.v\right|_{2} ^{2}}{2 \sigma_{s}^{2}}\right) .
\end{aligned}
$$

The underlining idea of the bilateral filtering is to do the smoothing according to pixels not only close in the space domain, but close in the feature domain as well, thus the edge sharpness is preserved by avoiding the cross edge smoothing. Bilateral filtering is closely related to other edge preserving techniques such as nonlinear diffusion and adaptive smoothing [12]. Bilateral filters are a nonlinear filter that smoothes the noise while preserving edge structures. The shiftvariant filtering operation of the bilateral filter is given by

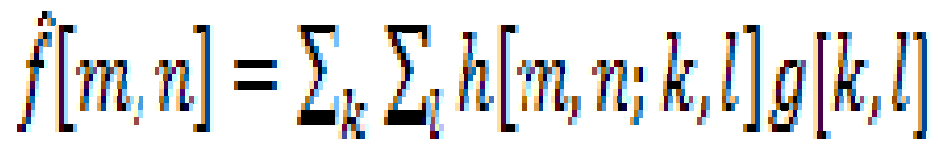

Where $f[m, n]$ is the restored image $h[m, n ; k, l]$, is the response at $[\mathrm{m}, \mathrm{n}]$ to an impulse at $[\mathrm{k}$, $1]$, and $\mathrm{g}[\mathrm{m}, \mathrm{n}]$ is the degraded image. For the Bilateral filter impulse response would be given as

$$
h\left[m_{0}, n_{0} ; m, n\right]= \begin{cases}r_{m_{0}, n_{0}}^{-1} \exp \left(-\frac{\left(m-m_{0}\right)^{2}+\left(n-n_{0}\right)^{2}}{2 \sigma_{d^{2}}{ }^{2}}\right) \exp \left(-\frac{\left(g[m, n]-g\left[m_{0}, n_{0}\right]\right)^{2}}{2 \sigma_{r}^{2}}\right), & {[m, n] \in \Omega_{m_{0}, n_{0}}} \\ 0, & \text { else }\end{cases}
$$

Where $\left[m_{0}, n_{0}\right]$ is the center pixel of the window, $\sigma_{\mathrm{d}}$ and $\sigma_{\mathrm{r}}$ are the standard deviations of the domain and range Gaussian filters, respectively, and

$$
\begin{aligned}
& r_{m_{0}, n_{0}}=\sum_{m=m_{0}-N}^{m_{0}+N} \sum_{n=n_{0}-N}^{n_{0}+N} \exp \left(-\frac{\left(m-m_{0}\right)^{2}+\left(n-n_{0}\right)^{2}}{2 \sigma_{d}^{2}}\right) \\
& x \exp \left(-\frac{\left(g[m, n]-g\left[m_{0}, n_{0}\right]\right)^{2}}{2 \sigma_{\mathrm{r}}{ }^{2}}\right)
\end{aligned}
$$


is a normalization factor that assures that the filter preserves average gray value in constant areas of the image. The edge-preserving denoising bilateral filter adopts a low pass Gaussian filter for both the domain filter and the range filter. The domain low pass Gaussian filter gives higher weight to pixels that are spatially close to the center pixel. The range low pass Gaussian filter gives higher weight to pixels that are similar to the center pixel in gray value. Combining the range filter and the domain filter, a bilateral filter at an edge pixel becomes an elongated Gaussian filter that is oriented along the edge. This ensures that averaging is done mostly along the edge and is greatly reduced in the gradient direction. This is the reason why the bilateral filter can smooth the noise while preserving edge structures. From a frequency domain perspective, bilateral filter is able to preserve edges while removing noise. On the other hand, the bilateral filter is essentially a smoothing filter. It does not sharpen edges. The edge rendered by the bilateral filter has the same level of blurriness as in the original degraded image, although the noise is greatly reduced. The results of the bilateral filtering are a significant improvement over a conventional linear low-pass filter. However, in order to enhance the sharpness of an image, we need to make some modifications to this filter.

\section{Adaptive-Bilateral Image projection}

In this section, we present a new sharpening and smoothing algorithm: the adaptive bilateral filter $(\mathrm{ABF})$. The response at $\left[m_{0}, n_{0}\right]$ of the proposed shift-variant ABF to an impulse at $[\mathrm{m}, \mathrm{n}]$ is Given by

$$
h\left[m_{0}, n_{0} ; m, n\right]= \begin{cases}r_{m_{0}, n_{0}}^{-1} \exp \left(-\frac{\left(m-m_{0}\right)^{2}+\left(n-n_{0}\right)^{2}}{2 \sigma_{d} d^{2}}\right) \exp \left(-\frac{\left(g[m, n]-g\left[m_{0}, n_{0}\right]-\zeta\left[m_{0}, n_{0}\right]\right)^{2}}{2 \sigma_{r}{ }^{2}}\right), & {[m, n] \in \Omega_{m_{0}, n_{0}}} \\ 0, & \text { else }\end{cases}
$$

(7)

and the normalization factor is given by

$$
\begin{aligned}
& r_{m_{0}, n_{0}}=\sum_{m=m_{n}-N} \sum_{n=n_{n}-N}^{m_{0}+N} \exp \left(-\frac{\left(m-m_{0}\right)^{2}+\left(n-n_{0}\right)^{2}}{2 \sigma_{d}^{2}}\right) \\
& \times \exp \left(-\frac{\left(g[m, n]-g\left[m_{0}, n_{0}\right]-\zeta\left[m_{0}, n_{0}\right]\right)^{2}}{2 \sigma_{T}{ }^{2}\left[m_{0}, m_{0}\right]}\right)
\end{aligned}
$$

Then in the overall Bilateral filter not much effect The ABF retains in general form with two modifications. First modification the offset $\zeta$ is introduced to the range filter, in the second modification both width of the range filter and $\zeta$ are locally adaptive in the ABF.. If $\zeta=0$ and $\sigma_{r}$ is fixed, the $\mathrm{ABF}$ will degenerate into a conventional bilateral filter. For the domain filter, a fixed low-pass Gaussian filter with $\sigma_{d}$ is adopted in the ABF. The combination of a locally adaptive $\zeta$ and $\sigma_{r}$ transforms the bilateral filter into a much more powerful filter that is capable of both smoothing and sharpening. Moreover when increasing the slope of the edge it increases image sharpens. To understand how the ABF works, we need to understand the role of $\zeta$ and $\sigma_{r}$ in the Adaptive Bilateral Filter the range filter can be interpreted as a 1-D filter that processes the histogram of the image. We will illustrate this viewpoint for the window of 
data enclosed in the box in the images. We index the images in the table by their [row, column] coordinates. For the conventional bilateral filter, the range filter is located on the histogram at the gray value of the current pixel and rolls off as the pixel values fall farther away from the center pixel value. By adding an offset $\zeta$ to the range filter, we are now able to shift the range filter on the histogram. As before, let $\Omega_{m_{0}, n_{0}}$ denote the set of pixels in the $(2 N+1) \times(2 N+1)$ window of pixels centered at $\left[m_{0}, n_{0}\right]$ Let MIN, MAX, and MEAN denote the operations of taking the minimum, maximum, and average value of the data in $\Omega_{\mathrm{m}_{0} \mathrm{n}_{0}}$ respectively. We will demonstrate the effect of bilateral filtering with a fixed domain Gaussian filter $\left(\sigma_{\mathrm{d}=1.0)}\right.$ and a range filter $\left(\sigma_{\mathrm{r}=} 20\right.$ ) shifted by the following choices for $\zeta$ :

1) No offset (conventional bilateral filter): $\zeta\left[m_{0}, n_{0}\right]=0$.

2) Shifting towards the MEAN $: \zeta\left[m_{0}, n_{0}\right]=-\Delta_{m_{0}, n_{0}}$

3) Shifting away from the MEAN $: \zeta\left[m_{0}, n_{0}\right]=\Delta_{m_{0}, n_{0}}$

4) Shifting away from the MEAN, to the MIN/MAX

$$
\begin{aligned}
& \zeta\left[m_{0}, n_{0}\right]-
\end{aligned}
$$

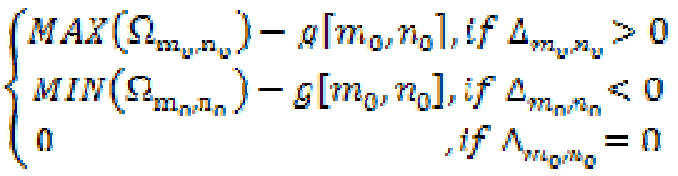

The parameter of the range filter controls the width of the range filter. It determines how selective the range filter is in choosing the pixels that are similar enough in gray value to be included in the averaging operation. If $\sigma_{\mathrm{r}}$ is large compared to the range of the data in the window, the range filter will assign similar weight to every pixel in the range. Then, it will not effect much on the overall bilateral filter. On the other hand, a small $\sigma_{\mathrm{r}}$ will make the range filter dominate the bilateral filter. The bilateral filtered image resembles the range filtered image when, and it resembles the domain filtered image when $\sigma_{\mathrm{r}=5}$ and it resembles the domain filtered image when $\sigma_{\mathrm{r}=50}$.

The pixel dependent offset $\zeta$ in the ABF is the key to slope restoration. With $\zeta$, we are able to restore the slope by transforming the local histogram of the image, thus circumventing the cumbersome process of locating edge normal and detecting edge profiles. Since at any pixel $\left[\mathrm{m}^{-}\right.$ $\left.{ }_{0}, n_{0}\right]$ in the image, the ABF output is bounded between $\operatorname{MIN}\left(\Omega_{m_{0}, n_{0}}\right)$ and $\operatorname{MAX}\left(\Omega_{m_{0}, n_{0}}\right)$. In general the $\mathrm{ABF}$ does not produce overshoot and undershoot. By making $\zeta$ and $\sigma_{\mathrm{r}}$ adaptive and jointly optimizing both parameters, we transform the bilateral filter into a much more powerful and versatile filter. To smooth the image at a given pixel, we can shift the range filter towards $\operatorname{MEAN}\left(\Omega_{\mathrm{m}_{Q_{0}} \mathrm{n}_{\Omega}}\right)$, and/or use a large $\sigma_{\mathrm{r}}$ which enables the spatial Gaussian filter to take charge of the bilateral filtering. To sharpen the image at a given pixel, we can shift the range filter away from the midpoint of the edge slope which will be approximately equal to MEAN

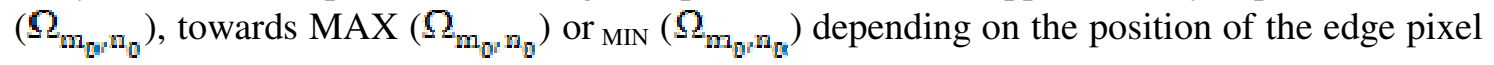
on the edge slope. At the same time, we would reduce $\sigma_{\mathrm{r}}$ accordingly. With a small $\sigma_{\mathrm{r}}$, the range filter dominates the bilateral filter and effectively pulls up or pushes down the pixels on the 
edge slope.

\section{Result Observation}

For the proposed approach a simulative model is carried out and the obtained result observation is as outlined below,

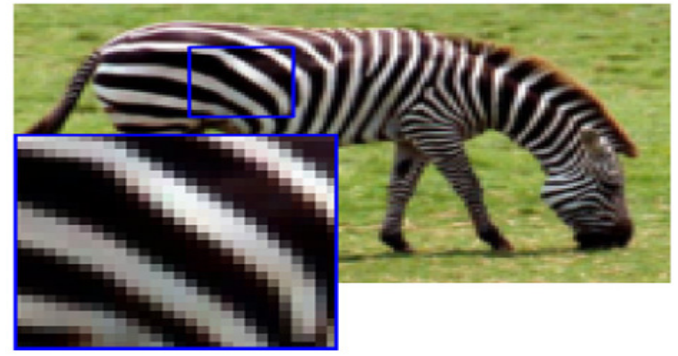

Figure1: interpolated image by Fourier interpolation without smoothening

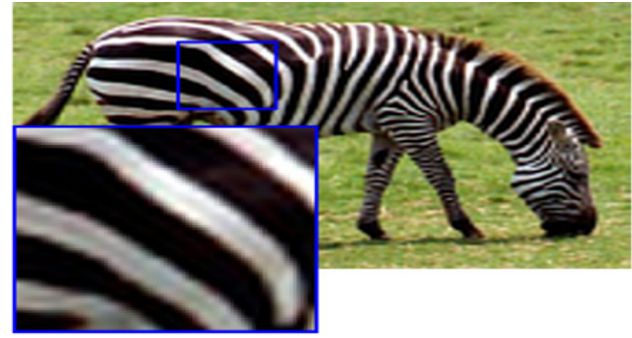

Figure2: interpolated image with bilateral filtration

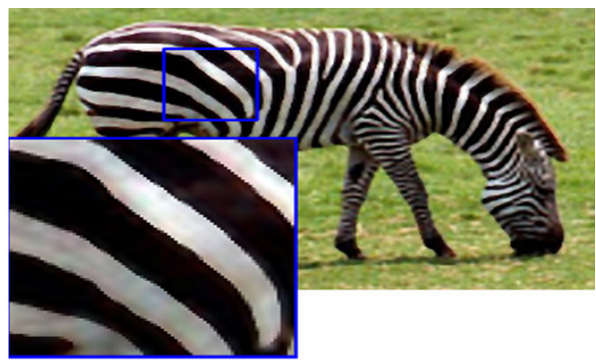

Figure3: interpolated image with Adaptive bilateral filtration

\section{Conclusion}

The quality of restored image is significantly improved compared with conventional bilateral filtration. In this paper it is observed that by using Adaptive filtration the ringing artifacts observed at the edges were eliminated while interpolating at the receiving side. The proposed adaptive bilateral filter contains two important modifications. First an offset $\zeta$ is introduced to range filter in $\mathrm{ABF}$, second both $\zeta$ and width of the range filter $\sigma_{\mathrm{r}}$ are locally adaptive.

with this approach the processing efficiency for image projection is improved. 
Advanced Computing: An International Journal ( ACIJ ), Vol.3, No.2, March 2012

\section{References}

[1] M. Irani and S. Peleg, "Motion analysis for image enhancement: resolution, occlusion and transparency," JVCIP, 1993.

[2] A.K. Katsaggelos, R. Molina, and J. Mateos, Super resolution of images and video, Synthesis Lectures on Image, Video, and Multimedia Processing. Morgan \& Claypool, 2007.

[3] J. Allebach and P. W. Wong, "Edge-directed interpolation," in ICIP, 1996.

[4] X. Li and M.T. Orchard, "New edge-directed interpolation,” IEEE Trans. on Image Processing, vol. 10 , no. 10 , pp. $1521-1527,2001$.

[5] B. S. Morse and D. Schwartzwald, "Image magnification using level set reconstruction," in CVPR, 2001.

[6] Y. Tai,W. Tong, and C. Tang, "Perceptually-inspired and edge-directed color image superresolution," in CVPR, 2006.

[7] S. Farisiu, M. D. Robinson, M. Elad, and P. Milanfar, "Fast and robust multiframe super resolution," IEEE Trans. on Image Processing, vol. 13, no. 10, pp. 1327-1344, 2004.

[8] S. Dai, M. Han, W. Xu, Y. Wu, and Y. Gong, "Soft edge smoothness prior for alpha channel super resolution," in CVPR, 2007.

[9] W. T. Freeman, T. R. Jones, and E. C. Pasztor, "Example-based super resolution,” IEEE Computer Graphics and Applications, 2002.

[10] D. Kong,M. Han,W. Xu, H. Tao, and Y. Gong, "Video super-resolution with scene-specific priors," in BMVC, 2006.

[11] C. Tomasi and R. Manduchi, "Bilateral filtering for gray and color images,” in ICCV, 1998.

[12] D. Barash, "A fundamental relationship between bilateral filtering, adaptive smoothing and the nonlinear diffusion equation,” IEEE Trans. on PAMI, vol. 24, no. 6, pp. 844-847, 2002.

[13] S. Baker and T. Kanade, "Limits on super-resolution and how to break them," IEEE Trans. on PAMI, vol. 24, no. 9, pp. 1167 - 1183, 2002.

[14] Z. Lin and H. Shum, "Fundamental limits of reconstruction based super-resolution algorithms under local translation,” IEEE Trans. on PAMI, vol. 26, no. 1, pp. 83 - 97, 2004.

[15] S. Saitoh, V. K. Tuan, and M. Yamamoto, “Convolution inequalities and applications," Journal of Inequalities in Pure and Applied Mathematics, vol. 4, no. 3, 2003.

\section{Short Biography}

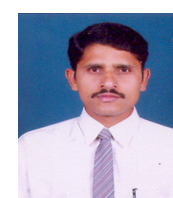

M. Nagaraju Naik received the B.Tech in Electronics and Communication Engineering from S.V.University, 1999, The Masters Degree in Digital Systems and Computer Electronics from JNTU Hyderabad. India. 2005. He is pursuing Ph.D in "Image Projection in 2-D and 3-D with higher resolution" from Andhra University College of Engineering (Autonomous), Vishakhapatnam, India. And his interests include Video Processing., Image Processing., and Signal Processing. 